\title{
SANS/WANS time-resolving neutron scattering studies of polymer phase transitions using NIMROD
}

Conference or Workshop Item

Accepted Version

Conference Proceeding

Mitchell, G. R., Bowron, D., Mateus, A., Bartolo, P.,

Gkourmpis, T., Phomphrai, K., Lopez, D. and Davis, F. (2013) SANS/WANS time-resolving neutron scattering studies of polymer phase transitions using NIMROD. In: MRS Fall Meeting 2012, 25-30 Nov 2012, Boston, MA. (MRS Online Proceedings Library, 1528, mrsf12-1528-vv06-04) Available at http://centaur.reading.ac.uk/31364/

It is advisable to refer to the publisher's version if you intend to cite from the work. See Guidance on citing.

Published version at: http://dx.doi.org/10.1557/opl.2013.363

All outputs in CentAUR are protected by Intellectual Property Rights law, including copyright law. Copyright and IPR is retained by the creators or other 
copyright holders. Terms and conditions for use of this material are defined in the End User Agreement.

\section{www.reading.ac.uk/centaur}

\section{CentAUR}

Central Archive at the University of Reading

Reading's research outputs online 
SANS/WANS Time-resolving Neutron Scattering Studiesof Polymer Phase Transitions Using NIMROD

Geoffrey Robert Mitchell ${ }^{1}$, Daniel Bowron ${ }^{2}$, Artur Mateus ${ }^{1}$, Paulo Bartolo ${ }^{1}$, Thomas Gkourmpis $^{3}$, Khamphee Phomphrai ${ }^{5}$, Daniel Lopez ${ }^{4}$, Fred Davis ${ }^{6}$

1 Centre for Rapid and Sustainable Product Development, Institute Polytechnic Leiria, Marinha Grande, Portugal;

2 ISIS, Science and Technology Facility Council, Didcot, United Kingdom; OX11 0QX

3 Innovation \& Technology, Borealis AB, Stenungsund SE-444 86 Sweden;

4 ICTP, Madrid, Spain;

5 Department of Chemistry, Mahidol University, Bangkok, Thailand.

6 Department of Chemistry, University of Reading UK RG6 6AD

\section{ABSTRACT}

We use new neutron scattering instrumentation to follow in a single quantitative time-resolving experiment, the three key scales of structural development which accompany the crystallisation of synthetic polymers. These length scales span 3 orders of magnitude of the scattering vector. The study of polymer crystallisation dates back to the pioneering experiments of Keller and others who discovered the chain-folded nature of the thin lamellae crystals which are normally found in synthetic polymers. The inherent connectivity of polymers makes their crystallisation a multiscale transformation. Much understanding has developed over the intervening fifty years but the process has remained something of a mystery. There are three key length scales. The chain folded lamellar thickness is $\sim 10 \mathrm{~nm}$, the crystal unit cell is $\sim 1 \mathrm{~nm}$ and the detail of the chain conformation is $\sim 0.1 \mathrm{~nm}$. In previous work these length scales have been addressed using different instrumention or were coupled using compromised geometries. More recently researchers have attempted to exploit coupled time-resolved small-angle and wide-angle x-ray experiments. These turned out to be challenging experiments much related to the challenge of placing the scattering intensity on an absolute scale. However, they did stimulate the possibility of new phenomena in the very early stages of crystallisation. Although there is now considerable doubt on such experiments, they drew attention to the basic question as to the process of crystallisation in long chain molecules. We have used NIMROD on the second target station at ISIS to follow all three length scales in a time-resolving manner for poly(e-caprolactone). The technique can provide a single set of data from 0.01 to $100 \AA-1$ on the same vertical scale. We present the results using a multiple scale model of the crystallisation process in polymers to analyse the results.

\section{INTRODUCTION}

Synthetic polymers which exhibit high degrees of stereoregularity are usually crystalline at room temperature. Polymers such as polyethylene, polypropylene, polycaprolactone and polyethylene terephthalate are examples with particular technological significance. The study of polymer crystallisation dates back to the pioneering experiments of Keller and others who in 1957 discovered the chain-folded nature of the thin lamellae crystals which are normally found in synthetic polymers [1]. Much understanding has been developed over the intervening fifty years but the process of primary nucleation has remained something of a mystery. Most recently time- 
resolved synchrotron-radiation based experiments have revealed the possibility of new phenomena in the very early stages of crystallization [2]. Although there is now considerable debate as to the meaning of such experiments, that draws attention to the basic question as to the process of crystallisation in long chain molecules and the mechanisms involved. For a simple liquid such as aluminium, it is straightforward to envisage a process by which individual atoms attach to a growth face. For a long chain molecule, in contrast, crystallisation involves conformational rearrangements as well as the development of precise atom positioning. It could be, as some have argued, that crystallisation is preceded by conformational changes, perhaps coupled to local density changes to yield an ordered, but non-crystalline structure which subsequently transforms in to crystals. This is the basis of the model of crystallisation process proposed by Strobl [3].

We have previously shown that precise quantitative structural information can be obtained from the broad Q neutron scattering data from disordered polymers through tight coupling of the scattering data and modelling techniques $[4,5]$ and we have initiated a programme to explore the possibilities afforded by the new data on an extended Q scale and perhaps most importantly on a single absolute scale for the differential scattering cross section available from NIMROD [6].

The advantage of the use of a pulsed neutron sources with crystallisation studies is the range of wavelengths used in the time-of flight procedures. NIMROD at the ISIS Pulsed neutron Source has a unique instrument specification, which exploits the longer wavelength neutrons available of the Target Station-2 to increase the upper limit of the accessible correlation lengths, while simultaneously extracting atomic resolution from the shorter wavelengths. NIMROD uses a large number of detectors to capture as many of the scattered neutrons as possible. The wavelength range is 0.04 to $10 \AA$. This gives a routine scattering vector range $|\mathrm{Q}|$ of 0.02 to $100 \AA^{-1}$, for some samples which give a strong small-angle scattering it may be possible to extend the useful $\mathrm{Q}_{\text {Min }}$ to $0.01 \AA^{-1}$ For the higher angle detectors, the resolution $\Delta \mathrm{Q} / \mathrm{Q} \sim 2 \%$. The instrument bridges the traditional gap between SANS and wide-angle neutron scattering, by using a common calibration procedure for all Q-scales. The data obtained from NIMROD will therefore enable the development of detailed and well constrained models of complex scattering systems.

The approach used here is to perform time-resolving neutron scattering experiments while the sample is subjected to a temperature jump from the melt to a crystallisation temperature. We select the crystallisation temperature so that crystallisation proceeds on a time scale which allows a series of data sets to be captured before crystallisation proceeds. We have specially designed a T jump stage to mount on NIMROD to take advantage of the large incident beam size, to maximise the flux, and thereby enhance the signal/noise ratio in the collected data.

\section{EXPERIMENT}

The neutron scattering data was obtained on the NIMROD diffractometer at the ISIS Pulsed Neutron facility. We used an incident beam size of $2 \times 2 \mathrm{~cm}$. The sample in the form of a $1 \mathrm{~mm}$ thickness film is sandwiched between vanadium foils mounted a copper frame with a $4 \mathrm{x} 4 \mathrm{~cm}$ aperture (the maximum beam size). This package is mounted at the centre of a large copper block through which fluid circulates to maintain the temperature as shown in Figure 1 


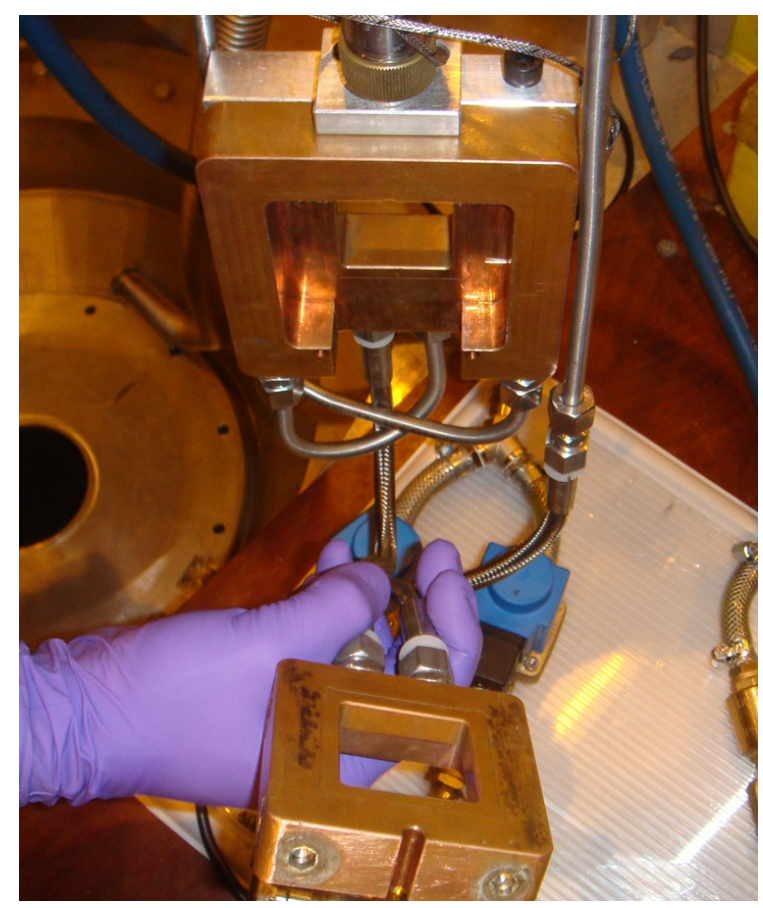

Figure 1. A photograph of the T Jump Stage mounted on a candlestick in the NIMROD Diffractometer

The result of the arrangement in Figure 1 is that the sample is held in a metallic can within a large mass of high thermal conductivity copper. Large capacity fluid baths containing an ethylene glycol/water mix are used to maintain the temperature of the sample. Electrically controlled valves are used to switch the flow from one fluid bath at the high temperature to the fluid from the low temperature bath. This results in a rapid temperature change without any chance of undercooling the temperature below the selected crystallization temperature. This is critical to this type of experiment.

Data from a number of polymer samples have been obtained. To obtain the best signal/noise ratio data we need to use per-deuterated polymers to avoid a large incoherent background fromany significant hydrogen content. $\mathrm{A}^{\mathrm{CH}_{2}}$ group in polymers such as polyethylene has a quite small scattering cross-section due to the almost exact cancelling of the negative scattering lengths of the hydrogen with the positive scattering length of the carbon. As a consequence the scattering arising from correlations between chain segments is low as is the scattering from chain folded crystalline lamellar. To an extent the requirement for per-deuterated material is a limitation to the technique, but we have successfully obtained data from fully hydrogenated polymers. The key test for our current work on time-resolved scattering experiments on semicrystalline polymers is the cycle time which yields useful data. Figure 2 shows some test data for a sample of deuterated poly( $\varepsilon$-caprolactone). 


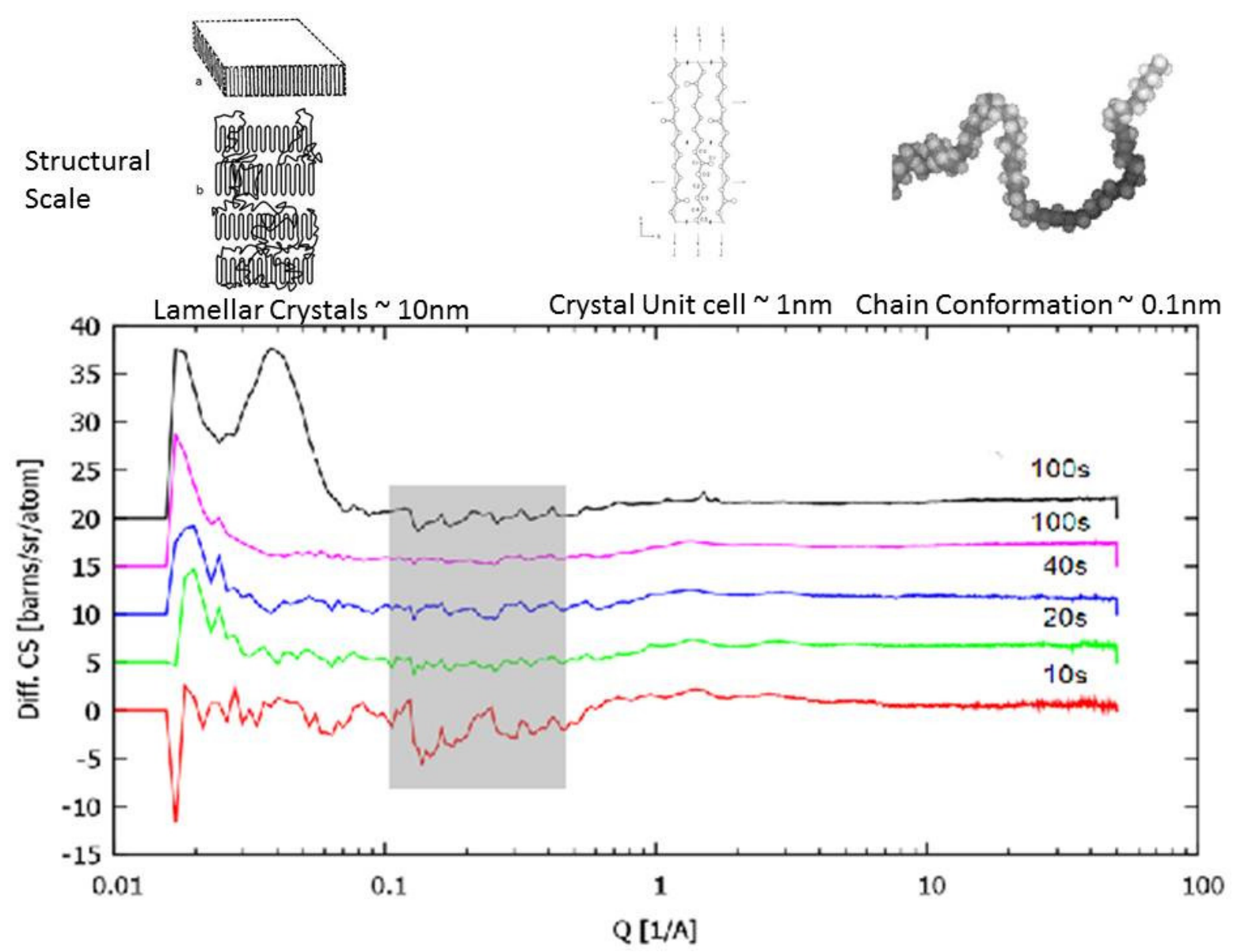

Figure 2. Experiment data for a sample of poly( $\varepsilon$-caprolactone) with the upper part showing the structural scale relevant to the Q region. Each curve shows the data accumulation time. The top curve (red) shows the data measured for a crystallised sample in 100s compared to the data obtain the melt in 100s (magenta). The three lower curves shows data obtained in the melt state for reduced times of 40,20 and 10s. The shaded area has a lower signal/noise

The data shown in Figure 2 reveals the striking scattering vector range of the data on NIMROD. The data is processed from its time-of-flight form to the differential scattering cross-sections using the GUDRUN package developed by the NIMROD team. This places all the data on an absolute scale over the complete scattering vector range. Here we have restricted the range to $|\mathrm{Q}|$ $\sim 50 \AA^{-1}$. Key to observe the crystallization process is the ability to see changes in the data from the scattering in the melt state. It is clear from the curves in Figure 2 that this can easily be done for data obtained with a 100s accumulation time. The top curve (black) shows data collected in 100s from a semi-crystalline sample. The remaining curves show data collected from the same melt state sample in a simple serial sequence of runs with different accumulation times. Data at a pulsed neutron source is usually integrated over a fixed value of the integrated proton current in terms of $\mu \mathrm{A}$ hrs which eliminates variations due to the disparity in the number of proton pulses delivered to the target. For a time-resolved experiment this is not helpful as it will lead to timeslices with different integration times. The data shown in Figure 2 was measured for a fixed 
accumulation time which may in some cases lead to noisy datasets due to the smaller number of

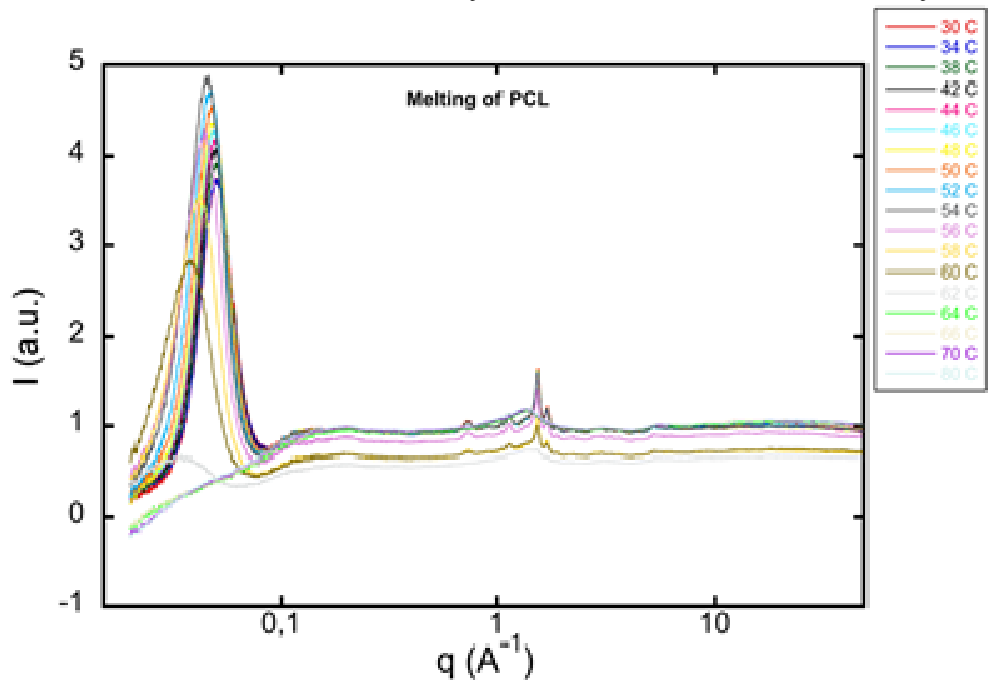

Figure 3 Experiment data for a sample of poly ( $\varepsilon$-caprolactone). Each curve shows the data obtained at constant temperature from room temperature to the melt state.

proton pulses delivered to the target and hence a lower intensity of the incident beam. Moreover, a shorter integration time may also reduce the number of detector outputs included in the data. For each run a statistical analysis is made of the outputs particularly in regions with a similiar Q range. Detectors for which the data is unacceptably noisy, which may be due to the reduced count time are eliminated from the integration process. This effect will be most severe at Q values where the scattered signal is low. As a consequence the noise is not simply related to the accumulation time or more specifically the number of events detected. An alternative approach is to record all events (ie neutron detection) with their time of arrival. In this mode called the 'Event Mode', we can integrate the events using time slices that are appropriate and for example produce datasets with constant signal/noise values. We will use this approach in future experiments. In the grey area, the signal to noise ratio is limited due to the reduced number of detectors which contribute data to this region. Recent upgrades to the instrument have now reduced this problem by increasing the detector coverage in this range by a factor of 5 . We anticipate this will also be possible with accumulation times of 20 or perhaps even 10 s especially where the signal is strong for example at low $Q$ values. Increasing the sample area, for example increasing the incident beam size to $2.5 \times 2.5 \mathrm{~cm}$ would increase the signal by $56 \%$, but would greatly amplify the challenge in providing a uniformity of temperature across the sample which is essential for the experiments described here.

Figure 3 shows the data obtained from poly ( $\varepsilon$-caprolactone d-6) at the constant temperature shown. At low $|\underline{Q}|$ the classic lamellar scattering is seen which arises from the chain folded lamellar crystals surrounded by amorphous materials. The sharp peaks at $|Q| \sim 1 \AA^{-1}$ is the equivalent to that seen using a laboratory diffractometer with a semi-crystalline polymer. The peaks arise from planes in the crystal lattice. The data at higher $\mathrm{Q}$ values provides information on the local chain conformation [3,4]. The data obtained in the melt state do not shown the lamellar scattering but do show a distinct broad peak at $|\mathrm{Q}| \sim 1 \AA^{-1}$ typical of molten polymers [4]. 


\section{CONCLUSION}

The new diffractometer opens up new possibilities which arise directly from the availability of high quality data on an absolute scale over an extended scattering vector range which embrace all the critical length scales from chain folded lamellar (10nm) to the fine details of the local chain conformation $(0.1 \mathrm{~nm})$ as well as the typical wide-angle scattering from semi-crystalline polymers $(1 \mathrm{~nm})$. The preliminary experiments we have performed show that time-resolving data can be obtained with a suitable time resolution for the study of phase transformations in polymer systems. The availability of neutron scattering data over an extended Q-range increases greatly the structural information over that obtain by the equivalent synchrotron based time-resolved Xray scattering experiments where the geometry compromises greatly the design of SAXS/WAXS experiments [7]. By combining data from both techniques, there is considerable promise in making progress in understanding the microscopic processes involved in the long-standing area of polymer crystallization.

\section{ACKNOWLEDGMENTS}

The neutron scattering was obtained at the STFC's ISIS Pulsed Neutron Source Facility Oxford shire UK. The work was supported by the FCT (Portugal).

\section{REFERENCES}

1. A.Keller Philos. Mag., Ser. 8, 2, 1171-1175 1957

2. EJ Heeley, AV Maidens, PD Olmsted, W Bras, IP Dolbnya, JPA Fairclough, NJ Terrill, and AJ Ryan, Macromolecules 36 (2003) 3656-3665

3. G.Strobl, Rev. Mod. Phys. 81, 12872009

4. G.R.Mitchell Neutron Diffraction to Soft Matter in Essentials of Neutron Techniques for Soft Matter Eds Imae, Toyoko et al 2011

5. G.R.Mitchell, B.Rosi and D.J.Ward Philos. Trans. Roy. Soc. (London) Series A 348 97-115 1994

6. T.Gkourmpis and G.R.Mitchell MRS Symposium 2012

7. G.R.Mitchell in 'Ecosustainable Polymer nanomaterials for food packaging' Eds C.Silvestre and S.Cimmino Taylor and Francis 2013 